\title{
Effect of Curcumin on the Formation of Epidural Fibrosis in an Experimental Laminectomy Model in Rats
}

\author{
Ozgur ISMAILOGLU', Zahir KIZILAY², Nesibe KAHRAMAN CETIN ${ }^{3}$, Abdullah TOPCU², Osman BERBER ${ }^{2}$ \\ ${ }^{1}$ Suleyman Demirel University, Faculty of Medicine, Department of Neurosurgery, Isparta, Turkey \\ ${ }^{2}$ Adnan Menderes University, Faculty of Medicine, Department of Neurosurgery, Aydin, Turkey \\ ${ }^{3}$ Adnan Menderes University, Faculty of Medicine, Department of Pathology, Aydin, Turkey
}

Corresponding author: Ozgur ISMAILOGLU ozguri_36@hotmail.com

\section{ABSTRACT}

AIM: To clarify the effects of topical application of curcumin on the prevention of epidural fibrosis.

MATERIAL and METHODS: Twenty-one rats were randomly divided into three equal groups (control, spongostan, local curcumin) and a laminectomy procedure was performed between T11 and L1 in all rats. Subsequently, spongostan soaked with curcumin (100 mg/kg) was applied topically. After four weeks, the vertebral column from T9 to L3, which included the paraspinal muscles and epidural scar tissue, was removed as a single piece and the epidural fibrosis and arachnoidal scarring were graded and histopathological analysis carried out accordingly. Kruskal-Wallis and Pearson Chi-Square tests were used for statistical analysis. A p-value of less than 0.05 was considered to be significant.

RESULTS: The grading of epidural fibrosis was far lower in the experimental group with curcumin compared to the control and spongostan groups, but the difference was not statistically significant.

CONCLUSION: The findings of this study show that local curcumin decreases the formation of epidural fibrosis and this effect of curcumin is thought to be mediated by reducing the functions of inflammatory cells such as macrophages, neutrophils and fibroblasts, and the anti-inflammatory and antioxidant effects.

KEYWORDS: Laminectomy, Epidural fibrosis, Failed back surgery, Curcumin, Rat

\section{INTRODUCTION}

$\mathrm{L}$ aminectomy is widely performed to access the epidural space for the treatment of spinal disorders. The healing Atissue may reduce an epidural scar at the laminectomy areas after performing the laminectomy (24). Epidural fibrosis (EF) related with failed back surgery (FBSS) may be defined as extensive fibrotic tissue formation at the epidural space, leading to extensive nerve root and dura mater adhesion (13). It has been reported that FBSS develops in $8-40 \%$ of the cases that underwent lumbar laminectomy. There are many factors in FBSS etiology; however, nearly $24 \%$ of these cases were due to EF (18). EF is a difficult field in spine surgery. Some studies related to this issue have been conducted and reported new methodologies like anti-inflammatory agents, fat graft, collagen membrane, Adcon-L, and fibrinolytic agents but no effective treatment option was found $(6,8,9,17,19,22)$. Curcumin (diferuloylmethane) gives turmeric its yellow color (the ground rhizome of Curcuma longa L.), and is often employed as a spice and is recognized for its anti-inflammatory features $(1,16)$. Curcumin affects the nuclear factor kappa $\beta$ (NF-KB) in pro-inflammatory cytokine reproduction, the antiapoptotic process, the functioning of leukocytes, and cell survival (1).

There is no information on how curcumin acts in EF. The objective of the present study was therefore to examine the impacts on EF after administering curcumin locally in a post laminectomy model.
Ozgur ISMAILOGLU (1) : 0000-0001-5183-5305

Zahir KIZILAY (D): 0000-0002-2021-0406

Nesibe KAHRAMAN CETIN (1) : 0000-0002-4549-1670
Abdullah TOPCU (1) : 0000-0001-8068-7006

Osman BERBER (i) : 0000-0003-0756-2631 


\section{MATERIAL and METHODS}

Adult female Wistar Albino rats that weighed $220-230$ grams were included in the experiment that was in line with the national institutes of health guidelines for care and use of laboratory animals. Approval was obtained from the local ethics committee on animal care (Aydin Adnan Menderes University, approval no: $6458310 / 2017 / 123$, date 28 November 2017).

\section{Experimental Groups}

Group 1: Control (C); T12 total laminectomy was carried out; nothing extra was applied, $\mathrm{n}=7$.

Group 2: Spongostan (S); T12 total laminectomy; distilled water and spongostan were applied on the laminectomy area, $\mathrm{n}=7$.

Group 3: Local curcumin (LC); T12 total laminectomy; 100 $\mathrm{mg} / \mathrm{kg}$ curcumin (purity_97) (Sigma Aldrich, Chemical Co., USA) with spongostan, $n=7$.

\section{Surgical Procedure and Preparation of Samples}

The temperature of the medium of the animals was adjusted as $22-25^{\circ}$ with average humidity and the light-dark cycle was adjusted as 12/12 hour. Food and water were given ad libitum. 50 mg/kg Ketamine hydrochloride $(\mathrm{HCl})$ (Ketalar, Parke-Davis, Turkey) and $10 \mathrm{mg} / \mathrm{kg}$ Xylazine $\mathrm{HCl}$ (Rompun, Bayer, Turkey) were administered intraperitoneally (IP) for anesthesia while spontaneously breathing. The temperature of the body was $37^{\circ} \mathrm{C}$ in the prone position. The rats' surgical areas were shaved and disinfected with povidone-iodine by the same surgeon. A midline skin incision was made on T10-L2. Another incision was made in the thoracolumbar fascia. The paravertebral tissues were dissected and the T10-L1 laminae were accessed. Total laminectomy was carried out at the T12 level for the rats in all groups while dura mater was visible and intact. Cotton pads were employed for hemostasis. The wounds were sutured with 5-0 polypropylene suture after application of topical curcumin. Under deep anesthesia, mixed xylazine $\mathrm{HCl}(10 \mathrm{mg} / \mathrm{kg} \mathrm{IP})$ and ketamine $\mathrm{HCl}(50 \mathrm{mg} / \mathrm{kg}$ IP) were administered on the $30^{\text {th }}$ day for cervical dislocation. T9 and L3 vertebral columns were removed en bloc.

\section{Histopathological Evaluation}

Vertebral columns T9-L3, paraspinal tissues and epidural scar tissue were excised en bloc, put into a container which was filled with $10 \%$ neutral buffered formalin solution and fixed. Decalcification was carried out in 7 days with $10 \%$ formic acid. The specimens were then taken from the laminectomy area. Tap water was used to wash them, and paraffin was used to embed the samples after routine tissue processing; 4-micron axial sections were taken. Hematoxylin-eosin and Masson's trichrome stains were used for staining. A blinded pathologist examined the histopathological sections and analyzed arachnoid involvement and EF grade. EF in the laminectomy area was assessed with the Olympus BX52 microscope after staining and was photographed by employing an Olympus DP 25 camera. The EF grading was analyzed in line with the scale of by $\mathrm{He}$ et al. (8). In addition, arachnoidal involvement was also examined.
Grade 0: No scar tissue on dura mater.

Grade 1: Only thin fibrous bands between scar tissue and dura mater.

Grade 2: Continuous adherence in less than $2 / 3$ of the laminectomy defect.

Grade 3: Large scar tissue adherence in more than $2 / 3$ of the laminectomy defect, or adherence extending to the nerve roots.

\section{Statistical Analysis}

The SPSS Package Program was employed for data analyses. To determine statistical significance for EF density, the KruskalWallis test was employed. For arachnoidal involvement, the exact $p$-value was computed and the outcomes were obtained in frequency. A p-value of less than 0.05 was considered to be significant.

\section{RESULTS}

\section{Complications Associated with Wound Recovery and Medical Practice}

No adverse effects of curcumin were seen in the wound area and the peripheral tissues.

\section{Histopathological Results}

The groups were graded in a histopathological manner in line with the He Scheme. Grade 3 (Figure 1A, B) and 2 EF were seen in six and one rats respectively in group $\mathrm{C}$. Grade 3 and 2 were seen in four and three rats respectively in group $S$. Grade 3 and 2 (Figure 2A, B) EF were seen in two and five rats respectively in group LC. These results showed that group LC was superior to group $\mathrm{C}$ and group $\mathrm{S}$ in reducing EF. However, there were no statistically significant differences $(p<0.097)$ (Figure 3).

The groups were also examined for arachnoid involvement. No statistically significant differences were detected $(p<0.734)$, (Figure 4).

\section{- DISCUSSION}

Inflammation is a complex defense reaction of endogenous or exogenous signals on vascularized tissues. Neutrophils, monocytes and macrophages have a role in the inflammation process. Proinflammatory cytokines such as interleukin (IL)1, IL-6, IL-8 and tumor necrosis factor alpha (TNF- $\alpha$ ) and the growth factors of platelet-derived growth factor (PDGF), transforming growth factor- $\beta 1$ (TGF- $\beta 1$ ), TGF- $\alpha$, insulin-like growth factor (IGF-1) and fibroblast growth factor (FGF) are produced in the injured area by the defense cells. Activated fibroblasts and epithelial cells function in the next step of healing by cleaning the waste material (5). Fibroblasts reproduce rapidly in response to activation of inflammatory cytokines and growth factors (TGF- $\beta$ and TGF- $\alpha$ ) in order to repair the local defective vertebral area where laminectomy is performed and reach the epidural space during spinal surgery (13). Fibroblasts transform into fibrocytes as a result of the production of collagen fibers. Scar tissue emerges from 


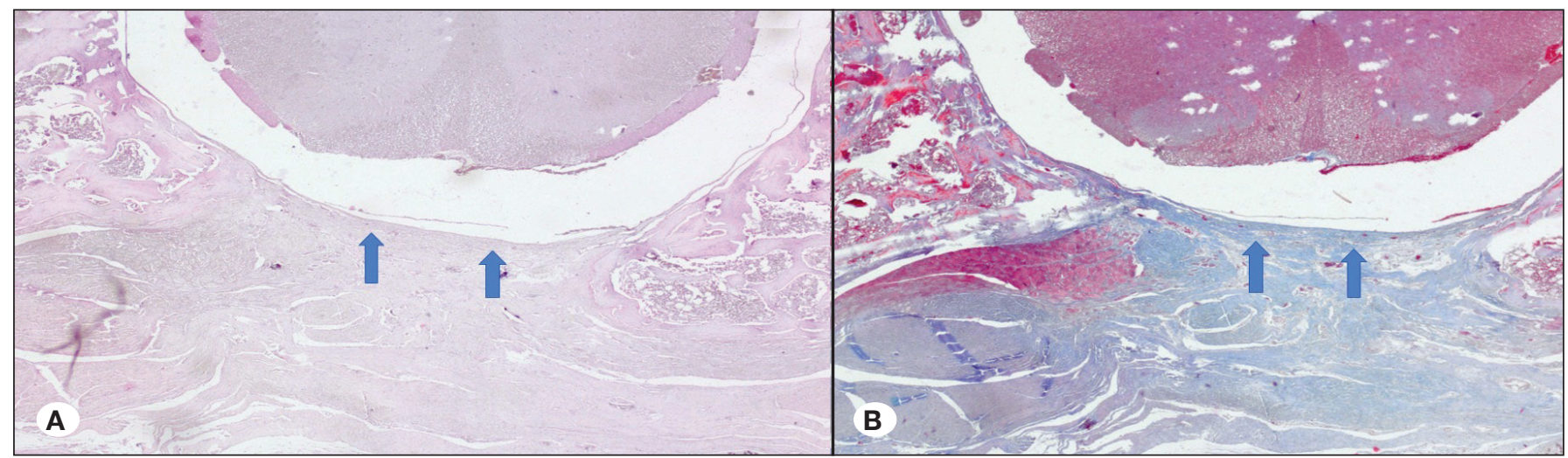

Figure 1: A) Hematoxylin-eosin (x40 magnification) staining for epidural fibrosis in the laminectomy sites. Grade 3 fibrosis in the control group: Scar tissue completely covered the laminectomy defects and adhered to the underlying dura mater (Arrows). B) Masson trichrome (x40 magnifications) staining for epidural fibrosis in the laminectomy sites. Grade 3 fibrosis in the control group: Scar tissue completely covered the laminectomy defects and adhered to the underlying dura mater (Arrows).

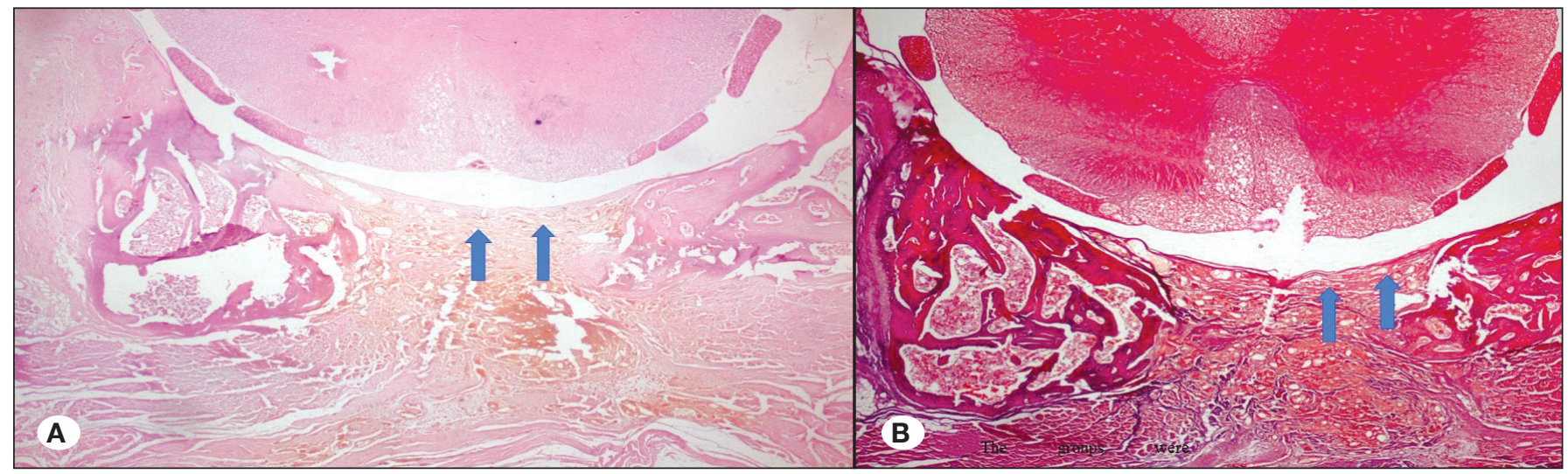

Figure 2: A) Hematoxylin-eosin (x40 magnification) staining for epidural fibrosis in the laminectomy sites. Grade 2 fibrosis in the curcumin group: Scar tissue adhered to the underlying dura mater and covered less than two-thirds of the laminectomy sites (Arrows). B) Masson trichrome ( $x 40$ magnifications) staining for epidural fibrosis in the laminectomy sites. Grade 2 fibrosis in the control group: Scar tissue adhered to the underlying dura mater and covered less than two-thirds of the laminectomy sites (Arrows).

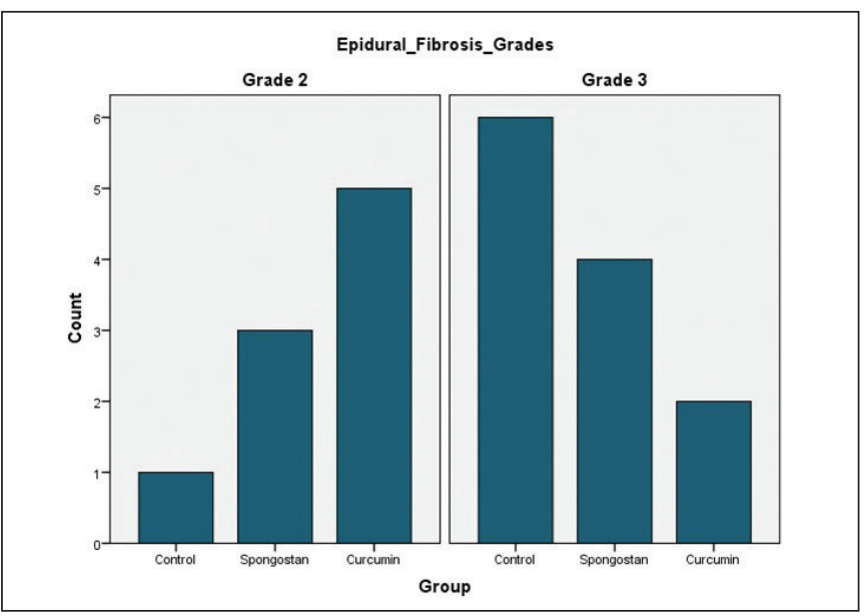

Figure 3: Histopathological assessment of epidural fibrosis grades of groups. There was no statistically significant difference between the groups ( $p>0.05)$. However, grade 2 epidural fibrosis was common in the curcumin group.

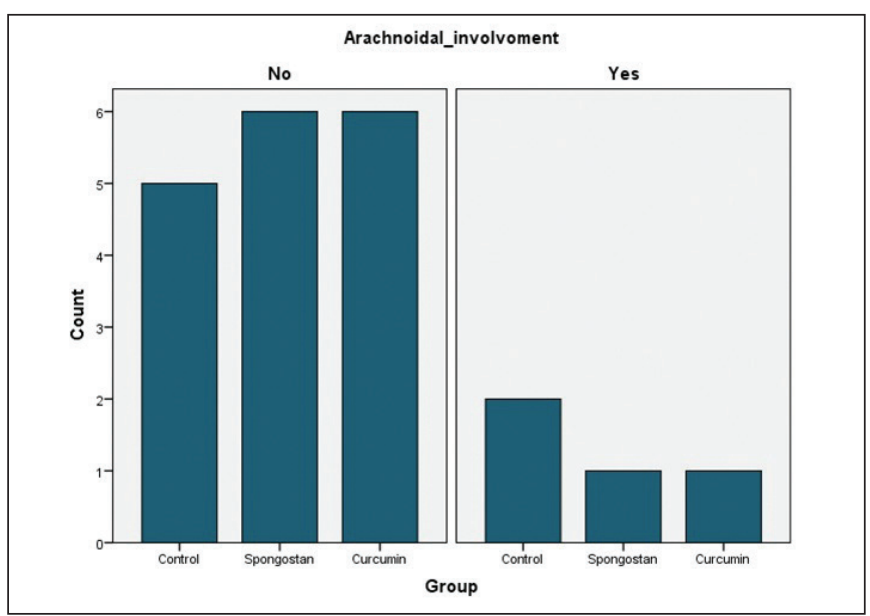

Figure 4: Frequency of arachnoidal involvement of the groups. There was no statistically significant difference between the groups ( $p>0.05)$. 
fibrous connective tissue. After this process, epidural fibrosis develops as a result of transformation of adipose tissue around dura mater into fibrous tissue (21).

Several clinical and laboratory studies have been conducted for avoidance of EF but satisfying results have not been reported yet $(9,14)$. Whatever the underlying reason, inflammatory responses and fibroblasts seem to be the most important factors during fibrosis. Unfortunately, there is no management modality other than anti-inflammatory drugs and immune suppressants to solve these problems. The aim of this study was to evaluate the effects of curcumin, which has anti-inflammatory properties, on EF.

Curcumin has antioxidant, anticancer, anti-apoptotic and antineurodegenerative properties (16). The impact of curcumin on EF after lumbar operations has not yet been investigated and this is the first study in the literature. As a result of this laboratory study, the Grade II EF rate in the curcumin group was found to be higher than Grade III in control group. Several effects of curcumin may have caused this result. Firstly, it might be the anti-inflammatory property of curcumin because curcumin blocks tissue proliferation and post traumatic tissue renewal by acting on macrophage cells with its antiinflammatory property (1). It is known that macrophages show these effects by increasing the function of factors such as TGF- $\beta$, TGF- $\alpha$, basic FGF, PDGF and vascular endothelial growth factor that increase proliferation and cell synthesis. Several studies suggest that curcumin decreases the amount of these factors (23). On the other hand, IL-6 and TGF- $\beta 1$ has been shown to be effective on EF (3). TNF- $\alpha$ has also been found to be another factor in EF (15). TNF- $\alpha$ especially increases the proliferation and differentiation of fibroblasts and the formation of a-smooth muscle actin and the extracellular matrix by transforming TGF- $\beta$; also, TNF- $\alpha$ and IL- 6 are strongly implicated as promoters of fibrosis $(3,15)$. Therefore, decreasing the effect of TNF- $\alpha$ and IL- 6 by using curcumin can result in a failure of fibroblast cell function. Curcumin has been found to have a suppressant effect on pro-inflammatory cytokine such as TNF- $\alpha$ which is a significant mediator in the inflammatory response $(1,7)$. Prior studies have also suggested that this mediator induces the amount of IL-6, prostaglandin E2 (PGE2), cyclooxygenase (COX-2) that have an important role in inflammation. Curcumin suppresses TNF-a-induced neuroinflammation IL-6, PGE2, COX-2 at a significant level (23). Moreover; curcumin has been shown to decrease post traumatic inflammation due to its anti-inflammatory properties in experimental spinal cord trauma studies (2). Spinal cord repair and neural function recovery were reported to be accelerated by curcumin via inhibition of glial scar formation and decreased inflammation (25). On the other hand, in the experimental study by Daishun Liu et. al., it was suggested that curcumin may decrease pulmonary fibrosis via inhibiting TGF- $\beta 2$ which drives the differentiation of lung fibroblasts to myofibroblasts (12). These studies strengthen the hypothesis that fibroblasts may be inhibited by curcumin and that it may therefore play a role in EF via decreasing inflammation. Our results therefore indicate that decrease in EF is due to the effect of curcumin on several steps of inflammation via its antiinflammatory properties.
Moreover; some studies suggest that curcumin, which has antioxidant effects, may inhibit nitric oxide and reactive oxygen species in macrophages. This suggestion supports an EF-decreasing effect of curcumin $(10,20)$.

The study also has some limitations. These are the inadequate number of rats, not comparing different doses of curcumin, and no comparison of the effect of the same dose with oral usage.

\section{CONCLUSION}

As a result of this study, the observation that the majority of the observed epidural fibrosis was grade II in the curcumin group and grade III in the control group indicates that curcumin has the potential to be used effectively in EF. Curcumin may decrease EF via anti-inflammatory mechanisms. However, further studies are needed to define which anti-inflammatory mechanisms play a role in this process.

\section{- REFERENCES}

1. Ali N, Soheil A: A review of therapeutic effects of curcumin. Current Pharmaceutical Design 19: 2032-2046, 2013

2. Bang WS, Kim KT, Seo YJ, Cho DC, Sung JK, Kim CH: Curcumin increase the expression of neural stem/progenitor cells and improves functional recovery after spinal cord injury. J Korean Neurosurg Soc 61(1):10-18, 2018

3. Battegay EJ, Raines EW, Colbert T, Ross R: TNF-alpha stimulation of fibroblast proliferation. Dependence on plateletderived growth factor (PDGF) secretion and alteration of PDGF receptor expression. J Immunol 154(11):6040-6047, 1995

4. Ceviz A, Arslan A, Ak HE, Inaloz S: The effect of the urokinase in preventing the formation of epidural fibrosis and/or leptomeningeal arachnoiditis. Surg Neurol 47(2):124-127,1997

5. Chin G, Diegelmann R, Schultz G: Cellular and molecular regulation of wound healing. In: Falabella A, Kirsner $R$ (eds). Wound Healing. New York: Taylor\&Francis, 2005:17-38

6. Da Costa RC, Pippi NL, Graca DL, Fialho SA, Alves A, Groff AC: The effect of the free fat graft or cellulose membrane implants on laminectomy membrane formation in dogs. Vet $\mathrm{J}$ 171:491-499, 2006

7. Gao X, Kuo J, Jiang H, et al: Immunomodulatory activity of curcumin: Suppression of lymphocyte proliferation, development of cell-mediated cytotoxicity, and cytokine production in vitro. Biochem Pharmacol 68(1):51-61, 2004

8. He Y, Revel M, Loty B: A quantitative model of postlaminectomy scar formation. Effects of a nonsteroidal antiinflammatory drug. Spine (PhilaPa 1976) 20(5):557-563, 1995

9. Ismailoglu O, Albayrak B, Gulsen I, Tanriover G, Demir N: Topical application of tacrolimus prevents epidural fibrosis in a rat post laminectomy model: Histopathological and ultrastructural analysis. Turk Neurosurg 21:630-633, 2011

10. Joe B, Lokesh BR: Role of capsaicin, curcumin and dietary $n-3$ fatty acids in lowering the generation of reactive oxygen species in rat peritoneal macrophages. Biochim Biophys Acta 1224(2):255-263, 1994 
11. LeHuec JC, Sadikki R, Cogniet A, Rigal J, Demezon H, Aunoble S: Role of the collagenmembrane in adhesion prevention strategy for complex spinal surgeries. Int Orthop 39(7):1383-1390, 2015

12. Liu D, Gong L, Zhu H, Pu S, Wu Y, Zhang W, Huang G: Curcumin inhibits transforming growth factor $\beta$ induced differentiation of mouse lung fibroblasts to myofibroblasts. Front Pharmacol 7:419, 2016

13. Lv P, Zhao J, Su W, Liang X, Zhang K: An experimental novel study: Hyperbaric oxygen treatment on reduction of epidural fibrosis via a down-regulation of collagen deposition, IL-6, and TGF- $\beta 1$. Eur J Orthop Surg Traumatol 25 Suppl 1:53-58, 2015

14. Mohi Eldin MM, Albed Razek NM: Epidural fibrosis after lumbar disc surgery: Prevention and outcome evaluation. Asian Spine J 9(3):370-385, 2015

15. Pierce GF, Mustoe TA, Lingelbach J, Masakowski VR, Griffin GL, Senior RM, et al: Platelet-derived growth factor and transforming growth factor-beta enhance tissue repair activities by unique mechanisms. J Cell Biol 109(1):429-440, 1989

16. Ramsewak RS, DeWitt DL, Nair MG: Cytotoxicity, antioxidant and anti-inflammatory activities of curcumins I-III from Curcuma longa. Phytomedicine 7(4): 303-308, 2000

17. Robertson JT, Maier K, Anderson RW, Mule JL, Palatinsky EA: Prevention of epidural fibrosis with ADCON-L in presence of a duratomy during lumbar disc surgery: Experiences with preclinical model. Neurol Res 21(1):S61-S66, 1999
18. Rodrigues FF, Dozza DC, de Oliveira CR, de Castro RG: Failed back surgery syndrome: Casuistic and etiology. Arq Neuropsiquiatr 64(3-B):757-761, 2006

19. Sandoval MA, Hernandez-Vaquero D: Preventing epidural fibrosis with nonsteroidal anti-inflammatory drugs. Eur Spine J 17(3):451-455, 2008

20. Sreejayan RM: Nitric oxide scavenging by curcuminoids. J Pharm Pharmacol 49(1):105-107, 1997

21. Sun Y, Wang L, Sun S, Liu B, Wu N, Cao X: The effect of 10-hidroxycamptothecine in preventing fibroblast proliferation and epidural scar adhesion after laminectomy in rats. Eur $\mathrm{J}$ Pharmacol 593:44-48, 2008

22. Turkoglu E, Dinc C, Tuncer C, Oktay M, Serbes G, Sekerci Z: Use of decorin to prevent epidural fibrosis in a postlaminectomy rat model. Eur J Pharmaco 724:86-91, 2014

23. Xiao L, Ding M, Fernandez A, Zhao P, Jin L, Li X: Curcumin alleviates lumbar radiculopathy by reducing neuroinflammation, oxidative stress and nociceptive factors. Eur Cell Mater 33:279-293, 2017

24. Zhang C, Kong X, Ning G, Liang Z, Qu T, Chen F, et al: Alltrans retinoic acid prevents epidural fibrosis through, NF-kB signaling pathway in post-laminectomy rats. Neuropharma 79:275-281, 2014

25. Zhao Z, Li X, Li Q: Curcumin accelerates the repair of sciatic nerve injury in rats through reducing Schwann cells apoptosis and promoting myelinization. Biomed Pharmacother 92:11031110, 2017 